\title{
COVID-19 PANDEMIC AND MENTAL HEALTH IN PAKISTAN
}

\author{
ALTAF QADIR KHAN \\ Department of Psychiatry, Postgraduate Medical Institute/Ameer ud Din Medical College/ \\ Lahore General hospital, Lahore
}

How to cite this article: Khan AQ. Covid-19: Covid-19 pandemic and mental health in Pakistan. Pak Postgrad Med J 2020;31(3): 121-122

This is an Open Access article distributed under the terms of the Creative Commons Attribution License (http://creativecommons.org/licenses/by/3.0), which permits unrestricted use, distribution, and reproduction in any medium, provided the original work is properly cited.

Correspondence to: Altaf Qadir Khan,

Professor, Department of Psychiatry,

Postgraduate Medical Institute/Ameer-ud-Din Medical

College/Lahore General Hospital, Lahore, Pakistan.

\section{Email: altafqadir@hotmail.com}

Mental Health problems and illnesses have accelerated at an alarming rate due to COVID-19 pandemic worldwide as well as in Pakistan with the potential of worsening due to crucial mismatch of available resources and burden of disease. Pakistan is the world's fifth most populous country with an estimated population of 220 million as of $2020^{1}$. With an increase in societal awareness and much needed de-stigmatization in recent years, increasing numbers of people are seeking help for common mental illnesses and the few studies that have been done in Pakistan have documented a much higher prevalence of mental health issues in Pakistan compared to Western countries. There are an estimated six million drug addicts in the country. Serious mental illnesses such as schizophrenia are estimated to have a prevalence rate of $1.5 \%$ in the population ${ }^{2}$. Child mental health problems also are common with a mean prevalence of $15 \%{ }^{3}$.

Increasing evidence points to the importance of mental health as a critical measure of overall health with more and more research emerging to show how mental and physical health are linked. Multiple studies have documented how untreated mental illness causes significantly worse outcomes in medical illness and conversely, how various medical illnesses can be a risk factor for, and worsen the prognosis for, mental illness. Neuropsychiatric and medical illnesses can thus no longer be viewed in isolation. Effective treatment of medical illnesses requires that equal attention be paid to neuropsychiatric (including mental) illness to reduce the functional burden of disease and prevent excess morbidity and mortality from medical illness.

Unfortunately, Mental Health has always been less prioritized by The Government than actually needed. Pakistan's public health budget is less than one percent of the country's GDP and mental health does not even have a separate budget. The World Health OrganizationAssessment Instrument for Mental Health Systems (WHO_AIMS) report on the mental health system in Pakistan published in $2009^{4}$ reported alarming deficiencies in financial, human and logistical resources. Only $0.4 \%$ of all health care expenditure by the government, was allocated for mental health, with 3729 outpatient mental health facilities, only five mental health hospitals, a total of 5,056 beds (2.7 beds per 100,00 population), and 342 psychiatrists (0.2 per 100,000 population). No significant amendments in government policy addressing mental health have been made since then.

The WHO declared COVID-19 a pandemic on 11th March 2020. Since then the number of cases worldwide have grown to $3,588,773$ with two lac forty seven thousand five hundred three $(247,503)$ deaths (as of 6th May 2020) $)^{5}$. Low income countries are particularly susceptible due to a severe shortage of healthcare resources.

The psychological effects on any community affected by COVID-19 illness can be broadly divided into three categories: the public, patients and healthcare workers (HCWs). In the public (uninfected people), a general sense of fear and uncertainty prevails (of being infected, of family members especially children, the elderly or the medically ill getting sick or dying), worsened by the constant media reports of new cases including deaths. While data specific for Pakistan does not yet exist, it is safe to say that the psychological burden will be significant due to a multitude of reasons: overcrowded and under resourced hospitals may get 
overwhelmed if cases spike; low literacy rates mean people have an incomplete understanding of the situation and may continue with casual life style despite warnings by healthcare workers and the government. Patients with the illness also undergo significant mental trauma and stress. Being infected with a viral illness with no approved 'cure', poorly understood modes of transmission and no lab tests to monitor daily progress causes a lot of anxiety for the patients ${ }^{6}$. The outbreak also has a severe psychological impact on the HCWs. HCWs, especially the ones caring for COVID 19 patients, report high levels of stress all across the world. Shortage of supplies including required medicines, personal protective equipment (PPE) and inadequate support are some factors contributing to the psychological burden of health care professionals ${ }^{7}$. They report symptoms of anxiety, depression, psychological distress and insomnia.

Despite the dire situation around the world, all is not doom and gloom in Pakistan. Pakistan's current case fatality ratio from COVID-19 illness is relatively low compared to many Western countries including the UK, USA and Italy ${ }^{8}$. The reasons for this are unclear at the moment but may include lower number of tests being performed and statistical under reporting of all-cause mortality as well as other, yet to be investigated factors including difference in virus strains, pre-existing cross immunity in the population etc. In additions, Tele-Health Centers and facilities are being practiced and established to compensate the out sourced Mental Health issues due to COVID-19 Pandemic ${ }^{9}$.

Conclusively, COVID-19 Pandemic not only posed a great challenge to the whole humanity especially healthcare providers but also emphasized on some less attended areas otherwise that is Mental Health and related issues. So far, Pakistan's public healthcare system, despite longstanding structural challenges has responded admirably to the threat of COVID-19 illness and it seems likely that with the continued efforts and progress, the future of Healthcare System especially Mental Healthcare will be much brighter in the twenty first century. To ensure this, following recommendations are proposed; Mental health must be provided a separate share in the national health budget commensurate with the existing needs of our population; psychiatric training centers need to be upgraded and enhanced nationally; all THQ and DHQ hospitals must have the services of a psychiatric department with consultant psychiatrists and affiliated mental health professionals working under their supervision available around the clock so patients and their families do not have to travel to large cities to access mental health services; all healthcare professionals and their families including doctors and nurses must be provided access to high quality mental health care round the clock for the duration of the COVID19 pandemic and beyond; a national media awareness campaign about mental health and mental illness should be started immediately to inform the public about these deadly illnesses and their prevention and treatment.

\section{REFERENCES}

1. Worldometers Pakistan Population counter live [internet] Cited 2020 April 25. Available from: https://www.worldometers.info/world-population/ pakistanpopulation/

2. Gadit AA, Khalid N. State of mental health in Pakistan: service, education \& research. Hamdard Foundation; 2002. Accessed from: https://jpma. org.pk/ PdfDownload/2680.

3. Mian A. Child and adolescent mental health in Pakistan. Adolescent Psychiatry. 2013;3(1):14-17. doi:10.2174/2210676611303010004

4. WHO-AIMS Report on Mental Health System in Pakistan [Internet]. World Health Organization, 2009. [cited 2020 April 25]. Available from: www.who.int/mental_health/pakistan who_aims_report.pdf

5. World Health Organization. Corona virus disease (COVID-19) Pandemic. https://www.who.int/ emergencies/diseases/novel-coronavirus-2019

[Accessed 2020 May 6.]

6. Ho CS, Chee CY, Ho RC. Mental health strategies to combat the psychological impact of COVID-19 beyond paranoia and panic. Ann Acad Med Singapore. 2020;49(1):1-3. Accessed from: https://www.ncbi.nlm. nih.gov/pubmed/32200399

7. Lai J, Ma S, Wang Y, Cai Z, Hu J, Wei N, et al. Factors associated with mental health outcomes among health care workers exposed to coronavirus disease 2019. JAMA Network Open. 2020;3(3):e203976. doi: 10.1001/jamanetworkopen.2020.3976

8. COVID-19 Map [Internet]. Johns Hopkins Coronavirus Resource Center. 2020 [cited 26 April 2020]. Available from: https://coronavirus.jhu.edu/map.html

9. Telemedicine centers to be set up in medical institutions: Governor Chaudhry Sarwar [Internet]. Thenews.com.pk. 2020 [cited 26 April 2020]. Available from: https://www.thenews.com.pk/print/ 632160-telemedicine-centres-to-beset-up-in-medicalinstitutions-governor-chaudhry-sarwar 